\title{
Increased Systemic Inflammation is Associated with Cardiac and Vascular Dysfunction over the First 12 Weeks of Antiretroviral Therapy among Undernourished, HIV-Infected Adults in Southern Africa
}

Michael Bestawros ${ }^{1}$, Takondwa Chidumayo ${ }^{2}$, Meridith Blevins ${ }^{3}$, Ashley Canipe ${ }^{4}$, Jay Bala ${ }^{5}$, Paul Kelly ${ }^{2,6}$, Suzanne Filteau ${ }^{7}$, Bryan E Shepherd ${ }^{3}$, Douglas C Heimburger ${ }^{5}$ and John R Koethe ${ }^{5,8^{*}}$

${ }^{1}$ Division of Cardiovascular Medicine, Vanderbilt University, Nashville, TN, USA

${ }^{2}$ University Teaching Hospital, Lusaka, Zambia

${ }^{3}$ Department of Biostatistics, Vanderbilt University, Nashville, TN, USA

${ }^{4}$ Division of Gastroenterology, Hepatology and Nutrition, Vanderbilt University, Nashville, TN, USA

${ }^{5}$ Vanderbilt Institute for Global Health, Nashville, TN, USA

${ }^{6}$ Barts and the London School of Medicine, London, UK

${ }^{7}$ London School of Hygiene \& Tropical Medicine, London, UK

${ }^{8}$ Division of Infectious Diseases, Vanderbilt University, Nashville, TN, USA

\begin{abstract}
Introduction: Persistent systemic inflammation is associated with mortality among undernourished, HIV-infected adults starting antiretroviral therapy (ART) in sub-Saharan Africa, but the etiology of these deaths is not well understood. We hypothesized that greater systemic inflammation is accompanied by cardiovascular dysfunction over the first 12 weeks of ART.

Methods: In a prospective cohort of 33 undernourished (body mass index $<18.5 \mathrm{~kg} / \mathrm{m}^{2}$ ) Zambian adults starting ART, we measured C-reactive protein (CRP), tumor necrosis factor- $\alpha$ receptor 1 (TNF- $\alpha$ R1), and soluble CD163 and CD14 at baseline and 12 weeks. An EndoPAT device measured the reactive hyperemia index (LnRHI; a measure of endothelial responsiveness), peripheral augmentation index (Al; a measure of arterial stiffness), and heart rate variability (HRV; a general marker of autonomic tone and cardiovascular health) at the same time points. We assessed paired changes in inflammation and cardiovascular parameters, and relationships independent of time point (adjusted for age, sex, and CD4+ T-cell count) using linear mixed models.
\end{abstract}

Results: Serum CRP decreased (median change $-3.5 \mathrm{mg} / \mathrm{l}, \mathrm{p}=0.02)$, as did TNF- $\alpha \mathrm{R} 1(-0.31 \mathrm{ng} / \mathrm{ml}, \mathrm{p}<0.01)$, over the first 12 weeks of ART. A reduction in TNF- $\alpha$ R1 over 12 weeks was associated with an increase in $\operatorname{LnRH}(p=0.03)$, and a similar inverse relationship was observed for CRP and $\mathrm{LnRHI}(p=0.07)$. Al increased in the cohort as a whole over 12 weeks, and a reduction in $\mathrm{sCD} 163$ was associated with a rise in the Al score $(p=0.04)$. In the pooled analysis of baseline and 12 week data, high CRP was associated with lower HRV parameters (RMSSD, $p=0.01$; triangular index, $p<0.01$ ), and higher TNF- $\alpha$ R1 accompanied lower HRV (RMSSD, $p=0.07$; triangular index, $p=0.06$ ).

Conclusions: Persistent inflammation was associated with impaired cardiovascular health over the first 12 weeks of HIV treatment among undernourished adults in Africa, suggesting cardiac events may contribute to high mortality in this population.

Keywords: Cardiovascular; HIV; Antiretroviral therapy; Nutrition; Sub-Saharan Africa; Inflammation

\section{Introduction}

Sub-Saharan Africa is disproportionately affected by the twin epidemics of HIV infection and chronic undernutrition, and a low body mass index (BMI) at the time of antiretroviral (ART) initiation is associated with increased early morbidity and mortality in HIV patients. In the first 90-180 days of ART, the mortality rate among undernourished HIV-infected adults is over $25 \%$ in some settings, which is 4 to 6 -fold higher than HIV-infected adults with normal BMI and is independent of CD4 T-cell suppression [1-4]. While the etiology of the high early mortality is likely multifactorial, persistent, heightened systemic inflammation is a major risk factor for death in undernourished, HIV-infected adults starting ART in the region $[5,6]$.

In individuals with normal BMI and without HIV, increased systemic inflammation is known to correlate with reduced heart rate variability, increased cardiovascular event rates, increased carotid intima-media thickness, coronary artery calcification, ischemic and dilated cardiomyopathies, myocardial infarction, and stroke [711]. We hypothesized that a similar link between inflammation and cardiovascular dysfunction, possibly compounded by undernutritionrelated factors such as reduced muscle mass and bioavailable phosphate stores, is present in malnourished, HIV-infected adults in sub-Saharan Africa and may contribute to the high rate of early ART mortality in these patients [12-16]. To investigate this question further, we conducted a prospective pilot study to assess relationships between circulating inflammatory markers and cardiovascular function among

*Corresponding author: John R Koethe, Division of Infectious Diseases, Vanderbilt University Medical Center, Tel: +1-615-322-2035; Fax: +1-615-343-6160; E-mail: john.r.koethe@vanderbilt.edu

Received January 05, 2015; Accepted February 13, 2015; Published February 21, 2015

Citation: Bestawros M, Chidumayo T, Blevins M, Canipe A, Bala J, et al (2015) Increased Systemic Inflammation is Associated with Cardiac and Vascular Dysfunction over the First 12 Weeks of Antiretroviral Therapy among Undernourished, HIV-Infected Adults in Southern Africa. J AIDS Clin Res 6: 431. doi:10.4172/2155-6113.1000431

Copyright: (c) 2015 Bestawros M, et al. This is an open-access article distributed under the terms of the Creative Commons Attribution License, which permits unrestricted use, distribution, and reproduction in any medium, provided the original author and source are credited. 
Citation: Bestawros M, Chidumayo T, Blevins M, Canipe A, Bala J, et al. (2015) Increased Systemic Inflammation is Associated with Cardiac and Vascular Dysfunction over the First 12 Weeks of Antiretroviral Therapy among Undernourished, HIV-Infected Adults in Southern Africa. J AIDS Clin Res 6: 431. doi:10.4172/2155-6113.1000431

Page 2 of 6

undernourished adults with advanced HIV over the first 12 weeks of ART.

\section{Methods}

The Mechanisms of Early Mortality in Adults Initiating Antiretroviral Therapy (MEMART) study was nested within the Nutritional Support for African Adults Starting Antiretroviral Therapy (NUSTART) study, which was a prospective, double-blinded, randomized placebocontrolled clinical trial designed to test whether the addition of vitamins and minerals to a lipid-based nutritional supplement would reduce early mortality in ART-eligible, undernourished, HIV-infected patients (trial registration \#PACTR201106000300631). The NUSTART study was conducted in Zambia and Tanzania from 2011 to 2013, and the MEMART pilot study prospectively enrolled a convenience sample of Zambian participants between August and December 2012 to evaluate the relationship between systemic inflammation and cardiovascular function.

Potentially eligible participants were evaluated by study staff at local health clinics and referred to University Teaching Hospital in Lusaka, Zambia for enrollment in the parent NUSTART trial and all study procedures. Inclusion criteria were a BMI $<18.5 \mathrm{~kg} / \mathrm{m}^{2}$, at least 18 years of age, ART- naïve (except for prior receipt of short-term Prevention of Mother To Child Transmission regimens), ART-eligible as determined by CD4+ T-cell count $<350$ cells/ $\mu$ l or Stage 3 or 4 HIV disease, willing to undertake intensive ART follow-up in the study clinic, and able to provide written or thumb print informed consent. Exclusion criteria were participation in a potentially conflicting research protocol, pregnancy by self-report, or the presence of an acute infectious condition aside from tuberculosis (participants with known tuberculosis needed to be on treatment) or oral candidiasis as assessed by study clinicians. All participants received efavirenz, tenofovir, and emtricitabine as a first-line ART regimen.

At baseline (prior to ART initation) and 12 weeks after ART initiation, measurements of systemic inflammation and oxidative stress were obtained. The inflammatory markers C-reactive protein (CRP), soluble CD163, and tumor necrosis factor- $\alpha$ receptor 1 (TNF- $\alpha$ R1, a soluble receptor of TNF- $\alpha$ that changes in relation to the circulating cytokine level) were measured using ELISA (R\&D Systems, Minneapolis, MN USA). Soluble CD14 was also measured using ELISA (Hycult Biotech, Uden, Netherlands). Urine samples were collected for assessment of oxidative stress using the 15-F2t-isoprostane/creatinine ratio (Oxford Biomedical Research, Rochester Hills, MI).

Cardiovascular assessments at baseline and after 12 weeks of ART included seated blood pressure and heart rate, ECGs to measure the corrected QT interval, and EndoPAT (Itamar Medical, Caesarea, Israel) assessment of the reactive hyperemia index (LnRHI; natural$\log$ transformed), peripheral augmentation index (AI), and heart rate variability (HRV, comprising the root mean square of the successive differences [RMSSD], triangular index, high and low frequency variation, and power ratio, which is the ratio of low-frequency to highfrequency oscillations) [17]. The EndoPAT is a portable device to assess peripheral arteriolar tonography, or the reactivity of digital vessels in response to a brief period of ischemia (produced by inflation of a blood pressure cuff) [18]. A LnRHI value below $\leq 0.51$ was previously shown to provides a sensitivity of $82 \%$ and a specificity of $77 \%$ for diagnosing coronary endothelial dysfunction in a large clinical study [18]. Higher AI values indicate increased arterial stiffness, as measured by the magnitude of the reflected arterial pressure wave during systole [19]. HRV, which was recorded for 5 minutes preceding brachial artery occlusion, is a measure of variations of both instantaneous heart rate and beat-to-beat intervals, and lower HRV is thought to reflect poor autonomic tone which confers increased risk of cardiovascular events and mortality [20,21].

Participant clinical and demographic characteristics were expressed as percentages or as median values with interquartile range. The change in each variable from recruitment to 12 weeks of ART was assessed using the Wilcoxon signed rank test. Our primary analysis evaluated whether changes in cardiovascular markers were associated with changes in inflammatory markers (i.e., within-patient paired comparison) using ordinary least squares regression, adjusted for the effect of baseline values. As a secondary analysis, we assessed the relationships between inflammatory biomarkers and cardiovascular markers irrespective of time point (baseline or 12 weeks) using a linear mixed model with a random effect for the patient to account for correlation that may arise from measurements taken from the same patient, adjusted for age, sex, and pre-treatment $\mathrm{CD} 4+\mathrm{T}$-cell count. Inflammatory markers were log-transformed, and cardiovascular markers remained on the unit scale (with the exception of LnRHI). Analyses were performed using $\mathrm{R}$ (version 2.12.1; www.r-project.org). Analysis scripts are posted at biostat.mc.vanderbilt.edu/ArchivedAnalyses.

The funding source had no role in the study design, data collection, data analysis, data interpretation, or writing of the report. The corresponding author had full access to all the data in the study and had the responsibility for the decision to submit for publication.

\section{Results}

Sixty ART-naïve adults were recruited for this study and 33 returned at 12 weeks for the clinical assessment and laboratory studies. Of the 27 patients without week 12 data, one-third $(n=9)$ had never started ART, 3 died, 3 withdrew due to relocation, pregnancy, or injury (road traffic accident), and 12 declined to return for week 12 measurements. Among the 3 deceased patients, one died of encephalitis and sepsis, and the other two died at home of unknown causes. Those included and excluded from the analysis were similar in regards to age, sex, and pre-treatment BMI, serum hemoglobin, and CD4+ T cell count ( $p>0.05$ for all). Among the 33 patients in the analysis cohort, 58\% were male, the median age was 36 years (interquartile range [IQR] 31-42 years), and the median CD4+ T cell count was 224 cells/ $\mu$ (IQR 151-273 cells/ $\mu$; Table 1). All participants were undernourished according to WHO criteria (i.e. BMI $<18.5 \mathrm{~kg} / \mathrm{m}^{2}$ ), with a median BMI of $16.7 \mathrm{~kg} / \mathrm{m}^{2}$ (IQR $15.4-17.6 \mathrm{~kg} / \mathrm{m}^{2}$ ). Over twelve weeks of ART, the median weight gain was $2.5 \mathrm{~kg}$ (IQR $0.1-6.0 \mathrm{~kg}$ ) and the median change in BMI was $0.9 \mathrm{~kg} /$ $\mathrm{m}^{2}\left(0.0-1.9 \mathrm{~kg} / \mathrm{m}^{2}\right)$. Baseline median CRP level was markedly elevated at $18.3 \mathrm{mg} / \mathrm{l}$ and declined to $6.4 \mathrm{mg} / \mathrm{l}$ by week $12(\mathrm{p}=0.02)$, and TNF- $\alpha$ $\mathrm{R} 1$ declined from 1.3 to $1.0 \mathrm{ng} / \mathrm{ml}(\mathrm{p}<0.01$, Table 1 and Figure 1$)$.

In the linear model of paired changes (Table 2), the change in TNF- $\alpha$ R1 over 12 weeks was inversely associated with the LnRHI score $(\mathrm{p}=0.03)$, and a similar relationship was observed for CRP $(\mathrm{p}=0.07)$, indicating increased endothelial responsiveness as inflammation declined. The change in sCD163 over 12 weeks was also inversely associated with the change in the AI score $(p=0.04)$, indicating increased arterial stiffness was present in those patients with greater reductions in sCD163. Lastly, a reduction in sCD163 and TNF- $\alpha$ R1 over 12 weeks was accompanied by a reduction in the resting heart rate $(\mathrm{p}=0.06$ for both). No other changes in the inflammatory markers were significantly associated with changes in the cardiovascular markers.

The associations between inflammatory markers and cardiovascular markers also were assessed using linear mixed models pooling data from 
Citation: Bestawros M, Chidumayo T, Blevins M, Canipe A, Bala J, et al. (2015) Increased Systemic Inflammation is Associated with Cardiac and Vascular Dysfunction over the First 12 Weeks of Antiretroviral Therapy among Undernourished, HIV-Infected Adults in Southern Africa. J AIDS Clin Res 6: 431. doi:10.4172/2155-6113.1000431

both time points and adjusting for age, sex, and baseline CD4+ T-cell count (Table 3). While this method does not assess whether biomarkers change in conjunction with each other, it detects relationships between biomarkers that may persist over time. CRP was inversely associated with several measurements of HRV: RMSSD $(\mathrm{p}=0.01)$, triangular index $(\mathrm{p}<0.01)$, and high frequency variations $(\mathrm{p}=0.02)$. There was also an inverse relationship between TNF- $\alpha$ R1 and systolic $(p=0.05)$ and diastolic blood pressure $(\mathrm{p}=0.05)$, RMSSD $(\mathrm{p}=0.07)$, and triangular

\begin{tabular}{|c|c|}
\hline \multicolumn{2}{|l|}{ Demographic and clinical characteristics } \\
\hline Female sex, $\mathrm{n}(\%)$ & $14(42 \%)$ \\
\hline Age (median years, IQR) & $36(31-42)$ \\
\hline Baseline BMI (median kg/m², IQR) & $16.7(15.4-17.6)$ \\
\hline Week 12 BMI (median kg/m², IQR) & $17.6(16.5-19.0)^{\mathrm{a}}$ \\
\hline Baseline CD4+ count (median cells/ $\mu \mathrm{l}, \mathrm{IQR}$ ) & $224(151-273)$ \\
\hline Week 12 CD4+ count (median cells/ $\mu$ I, IQR) & $349(275-426)^{a}$ \\
\hline
\end{tabular}

Median serum, urine, and cardiovascular values pre-treatment and after 12 weeks of antiretroviral therapy, median (IQR)

\begin{tabular}{|c|c|c|}
\hline Variable & Recruitment & Week 12 of ART \\
\hline C-reactive protein (mg/l) & $18.3(4.5-64.8)$ & $6.4(3.8-19.7)^{a}$ \\
\hline TNF- $\alpha$ receptor $1(\mathrm{ng} / \mathrm{ml})$ & $1.3(0.8-2.4)$ & $1.0(0.6-1.3)^{\mathrm{a}}$ \\
\hline Soluble CD163 (ng/ml) & $684(511-1022)$ & $531(376-1040)$ \\
\hline Soluble CD14 $(\mu \mathrm{g} / \mathrm{ml})$ & $2.4(2.1-2.7)$ & $2.5(2.0-3.1)$ \\
\hline Isoprostane-to-creatinine ratio & $0.02(0.01-0.05)$ & $0.09(0.05-0.24)^{a}$ \\
\hline Augmentation Index & $11.9(1.0-30.0)$ & $19.0(0.1-25.9)$ \\
\hline Reactive Hyperemia Index & $0.6(0.3-0.8)$ & $0.6(0.4-0.7)$ \\
\hline QTc (ms) & $434(421-447)$ & $431(417-446)$ \\
\hline RMSSD (ms) & $26.4(17.5-41.3)$ & $37.2(19.2-71.6)^{a}$ \\
\hline Triangular Index & $6.8(5.9-10.6)$ & $9.6(6.1-14.5)$ \\
\hline Low frequency variations $\left(\mathrm{ms}^{2}\right)$ & $130.2(85.0-151.9)$ & $112.9(76.1-151.6)$ \\
\hline High frequency variations $\left(\mathrm{ms}^{2}\right)$ & $214.7(154.7-260.8)$ & $232.6(169.7-327.1)$ \\
\hline Power Ratio & $0.6(0.4-0.9)$ & $0.4(0.3-0.9)$ \\
\hline Systolic blood pressure $(\mathrm{mm} \mathrm{Hg})$ & $100(93-110)$ & $110(100-120)^{a}$ \\
\hline Diastolic blood pressure $(\mathrm{mm} \mathrm{Hg})$ & $60(60-70)$ & $70(60-70)$ \\
\hline Heart rate $(\mathrm{bpm})$ & $78(71-89)$ & $68(63-82)$ \\
\hline
\end{tabular}

Abbreviation: BMI: Body Mass Index; QTC: Corrected QT interval; RMSSD: Root Mean Square of the Successive Differences; TNF- $\alpha$ : Tumor Necrosis Factor- $\alpha$. Reactive hyperemia index is natural log transformed a: $p<0.05$ for change at 12 weeks

Table 1: Description of the cohort $(n=33)$. index $(\mathrm{p}=0.06)$. However, TNF- $\alpha$ R1 was positively associated with LnRHI $(p=0.01)$, which was unexpected given the directionality of relationships observed in the linear models of paired changes in inflammatory and cardiovascular variables. Higher heart rate was associated with higher CRP $(\mathrm{p}<0.01)$ and soluble CD14 levels $(\mathrm{p}<0.01)$. While there was a significant increase in the urine isoprostane/ creatinine ratio over 12 weeks, the ratio was not associated with any of the inflammatory biomarkers or with the cardiovascular parameters (data not shown). We did not detect any other significant relationships between inflammatory biomarkers and cardiovascular markers.

\section{Discussion}

This pilot study examining relationships between inflammatory and cardiovascular markers in undernourished HIV-infected adults during initiation of ART demonstrated several novel findings that may implicate poor cardiovascular health and function as one of the mechanistic links in the well-described relationship between persistent inflammation and early mortality in this population. We found that higher levels of circulating inflammatory markers were associated with reduced vascular endothelial responsiveness and heart rate variability, both indicative of poor cardiovascular health, at baseline and after 12 weeks of ART. However, we did not observe paired changes in several of these variables, which may have been due in part to our small cohort size. Based on these findings, further studies are warranted to assess the relationship between inflammation and cardiovascular health over time, and to assess whether cardiovascular dysfunction, particularly sudden cardiac death, is a frequent proximate cause of early mortality among malnourished adults starting ART.

Serum CRP levels in our participants were high at baseline (median $18.3 \mathrm{mg} / \mathrm{l})$ and remained elevated, although reduced, at 12 weeks (6.4 $\mathrm{mg} / \mathrm{l})$. While CRP levels over $3.0 \mathrm{mg} / \mathrm{l}$ have been associated with high cardiovascular risk in US and European studies of HIV-infected and uninfected adults, caution is warranted in extrapolating these findings to African HIV patients given differences in populations, background disease risk, and laboratory methodology [22]. During 6 years of observation, Triant et al. found that the odds of an acute myocardial infarction was more than four-fold higher for patients with both an elevated CRP and HIV compared to uninfected individuals with normal CRP [23]. During 29 months of observation, Duprez et al. similarly found that the odds of a cardiovascular event were two-fold

\begin{tabular}{|c|c|c|c|c|c|c|c|c|c|c|c|c|}
\hline \multirow[b]{2}{*}{$\begin{array}{l}\text { Inflammation } \\
\text { biomarkers } \\
\text { (change in log- } \\
\text { transformed } \\
\text { regression predictor) }\end{array}$} & \multicolumn{12}{|c|}{ Cardiovascular markers (change in regression outcome) } \\
\hline & 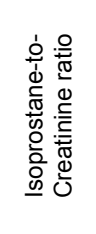 & 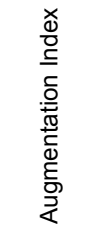 & 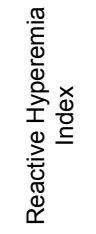 & $\frac{0}{0}$ & $\sum_{\substack{\infty \\
\mathscr{D}}}^{\mathscr{D}}$ & 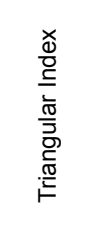 & 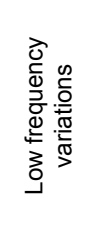 & 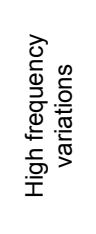 & 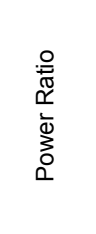 & 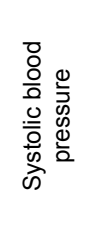 & 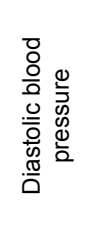 & 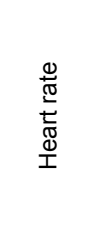 \\
\hline C-reactive protein & $\begin{array}{c}0.00 \\
p=0.96\end{array}$ & $\begin{array}{l}-2.22 \\
p=0.54\end{array}$ & $\begin{array}{l}-0.09 \\
p=0.07\end{array}$ & $\begin{array}{c}1.35 \\
p=0.74\end{array}$ & $\begin{array}{l}-5.76 \\
p=0.37\end{array}$ & $\begin{array}{l}-1.32 \\
p=0.18\end{array}$ & $\begin{array}{c}5.60 \\
p=0.65\end{array}$ & $\begin{array}{l}-22.80 \\
p=0.18\end{array}$ & $\begin{array}{c}0.05 \\
p=0.81\end{array}$ & $\begin{array}{l}1.62 \\
p=0.60\end{array}$ & $\begin{array}{l}-0.09 \\
p=0.97\end{array}$ & $\begin{array}{c}3.43 \\
p=0.23\end{array}$ \\
\hline $\begin{array}{l}\text { Tumor necrosis factor- } \alpha \\
\text { receptor } 1\end{array}$ & $\begin{array}{l}-0.08 \\
p=0.52\end{array}$ & $\begin{array}{c}-6.22 \\
p=0.54\end{array}$ & $\begin{array}{l}-0.26 \\
\mathbf{p}=\mathbf{0 . 0 3}\end{array}$ & $\begin{array}{c}9.62 \\
p=0.31\end{array}$ & $\begin{array}{l}-10.09 \\
p=0.69\end{array}$ & $\begin{array}{l}-2.13 \\
p=0.41\end{array}$ & $\begin{array}{l}12.78 \\
p=0.70\end{array}$ & $\begin{array}{l}-38.72 \\
p=0.40\end{array}$ & $\begin{array}{c}0.02 \\
p=0.97\end{array}$ & $\begin{array}{c}0.20 \\
p=0.98\end{array}$ & $\begin{array}{l}-3.98 \\
p=0.38\end{array}$ & $\begin{array}{l}14.10 \\
p=0.06\end{array}$ \\
\hline Soluble CD163 & $\begin{array}{c}0.02 \\
p=0.86\end{array}$ & $\begin{array}{l}-14.06 \\
\mathbf{p}=\mathbf{0 . 0 4}\end{array}$ & $\begin{array}{l}-0.11 \\
p=0.17\end{array}$ & $\begin{array}{c}2.71 \\
p=0.68\end{array}$ & $\begin{array}{l}-2.05 \\
p=0.91\end{array}$ & $\begin{array}{l}-1.75 \\
p=0.34\end{array}$ & $\begin{array}{l}28.46 \\
p=0.16\end{array}$ & $\begin{array}{c}1.86 \\
p=0.95\end{array}$ & $\begin{array}{c}0.33 \\
p=0.23\end{array}$ & $\begin{array}{l}5.19 \\
p=0.30\end{array}$ & $\begin{array}{c}2.34 \\
p=0.46\end{array}$ & $\begin{array}{c}9.44 \\
p=0.06\end{array}$ \\
\hline Soluble CD14 & $\begin{array}{l}-0.03 \\
p=0.83\end{array}$ & $\begin{array}{l}-15.37 \\
p=0.25\end{array}$ & $\begin{array}{l}-0.05 \\
p=0.77\end{array}$ & $\begin{array}{c}-4.08 \\
p=0.73\end{array}$ & $\begin{array}{l}11.25 \\
p=0.76\end{array}$ & $\begin{array}{c}0.41 \\
p=0.91\end{array}$ & $\begin{array}{c}9.91 \\
p=0.79\end{array}$ & $\begin{array}{l}-21.07 \\
p=0.72\end{array}$ & $\begin{array}{l}-0.03 \\
p=0.96\end{array}$ & $\begin{array}{l}-2.51 \\
p=0.78\end{array}$ & $\begin{array}{c}2.45 \\
p=0.67\end{array}$ & $\begin{array}{l}13.31 \\
p=0.17\end{array}$ \\
\hline
\end{tabular}

Models estimate the relationship between the change in log-transformed inflammation biomarker and change in cardiovascular marker using ordinary least squares regression, adjusted for baseline values. Bold values represent statistically significant findings.

Abbreviation: QTC: Corrected QT interval; RMSSD: Root Mean Square of the Successive Differences.

Reactive hyperemia index is natural log transformed 
Citation: Bestawros M, Chidumayo T, Blevins M, Canipe A, Bala J, et al. (2015) Increased Systemic Inflammation is Associated with Cardiac and Vascular Dysfunction over the First 12 Weeks of Antiretroviral Therapy among Undernourished, HIV-Infected Adults in Southern Africa. J AIDS Clin Res 6: 431. doi:10.4172/2155-6113.1000431

Page 4 of 6

\begin{tabular}{|c|c|c|c|c|c|c|c|c|c|c|c|c|}
\hline \multirow[b]{2}{*}{$\begin{array}{l}\text { Inflammation } \\
\text { biomarkers } \\
\text { (log-transformed } \\
\text { regression predictor) }\end{array}$} & \multicolumn{12}{|c|}{ Cardiovascular markers (regression outcome) } \\
\hline & 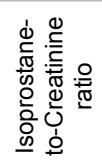 & 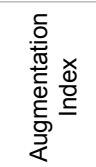 & 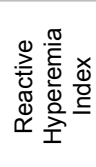 & $\frac{0}{0}$ & $\begin{array}{l}\text { O } \\
\sum_{i=1}^{\infty}\end{array}$ & 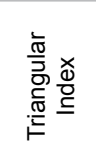 & 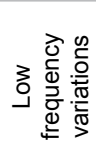 & 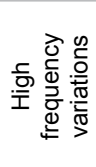 & 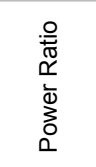 & 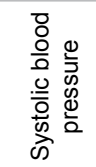 & 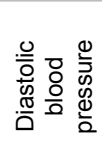 & 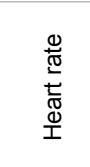 \\
\hline C-reactive protein & $\begin{array}{c}0.01 \\
p=0.95\end{array}$ & $\begin{array}{c}-1.36 \\
p=0.06\end{array}$ & $\begin{array}{c}0.02 \\
p=0.37\end{array}$ & $\begin{array}{l}-0.77 \\
p=0.90\end{array}$ & $\begin{array}{c}-7.03 \\
\mathbf{p}=\mathbf{0} .01\end{array}$ & $\begin{array}{l}-1.73 \\
p<0.01\end{array}$ & $\begin{array}{c}-0.32 \\
p=0.86\end{array}$ & $\begin{array}{l}-26.35, \\
\mathbf{p}=\mathbf{0 . 0 2}\end{array}$ & $\begin{array}{c}0.18, \\
p=0.18\end{array}$ & $\begin{array}{c}-2.51 \\
\mathrm{p}=0.28\end{array}$ & $\begin{array}{l}-1.93 \\
p=0.16\end{array}$ & $\begin{array}{c}4.90 \\
\mathbf{p}<0.01\end{array}$ \\
\hline $\begin{array}{l}\text { Tumor necrosis factor- } \alpha \\
\text { receptor } 1\end{array}$ & $\begin{array}{l}-0.07 \\
p=0.51\end{array}$ & $\begin{array}{c}0.44 \\
p=0.95\end{array}$ & $\begin{array}{c}0.14 \\
p=0.01\end{array}$ & $\begin{array}{l}1.30 \\
p=0.77\end{array}$ & $\begin{array}{l}-17.83 \\
p=0.07\end{array}$ & $\begin{array}{l}-2.12 \\
p=0.06\end{array}$ & $\begin{array}{l}-12.02 \\
p=0.34\end{array}$ & $\begin{array}{l}-19.54 \\
p=0.40\end{array}$ & $\begin{array}{l}-0.04 \\
p=0.96\end{array}$ & $\begin{array}{l}-9.40 \\
p=0.05\end{array}$ & $\begin{array}{l}-5.12 \\
p=0.05\end{array}$ & $\begin{array}{l}7.70 \\
p=0.11\end{array}$ \\
\hline Soluble CD163 & $\begin{array}{l}-0.11 \\
p=0.20\end{array}$ & $\begin{array}{l}-6.01 \\
p=0.40\end{array}$ & $\begin{array}{l}-0.04 \\
p=0.46\end{array}$ & $\begin{array}{r}0.02 \\
\mathrm{p}=0.70\end{array}$ & $\begin{array}{c}5.75 \\
p=0.39\end{array}$ & $\begin{array}{l}-0.79 \\
p=0.60\end{array}$ & $\begin{array}{l}11.60 \\
p=0.23\end{array}$ & $\begin{array}{l}-20.80 \\
p=0.33\end{array}$ & $\begin{array}{c}0.25 \\
p=0.18\end{array}$ & $\begin{array}{c}2.06 \\
\mathrm{p}=0.26\end{array}$ & $\begin{array}{c}0.70 \\
p=0.34\end{array}$ & $\begin{array}{r}4.82 \\
\mathrm{p}=0.21\end{array}$ \\
\hline Soluble CD14 & $\begin{array}{c}0.00 \\
p=0.95\end{array}$ & $\begin{array}{l}-13.88 \\
p=0.10\end{array}$ & $\begin{array}{c}-0.04 \\
p=0.67\end{array}$ & $\begin{array}{c}6.62 \\
p=0.19\end{array}$ & $\begin{array}{l}12.34 \\
p=0.43\end{array}$ & $\begin{array}{l}-1.70 \\
p=0.46\end{array}$ & $\begin{array}{l}-5.34 \\
p=0.78\end{array}$ & $\begin{array}{l}-46.00 \\
p=0.24\end{array}$ & $\begin{array}{c}0.19 \\
p=0.58\end{array}$ & $\begin{array}{l}-9.04 \\
p=0.17\end{array}$ & $\begin{array}{l}-5.53 \\
p=0.11\end{array}$ & $\begin{array}{l}15.52 \\
\mathbf{p}<0.01\end{array}$ \\
\hline
\end{tabular}

Results from linear mixed models pooling data across both recruitment and 12 weeks of ART. Models adjusted for age, sex, and baseline CD4+ T-cell count and included a random effect for patient. Bold values represent statistically significant findings.

Abbreviation: QTC: Corrected QT interval; RMSSD: Root Mean Square of the Successive Differences

Reactive hyperemia index is natural $\log$ transformed

Table 3: Regression coefficients estimating the associations between cardiovascular markers and inflammation.
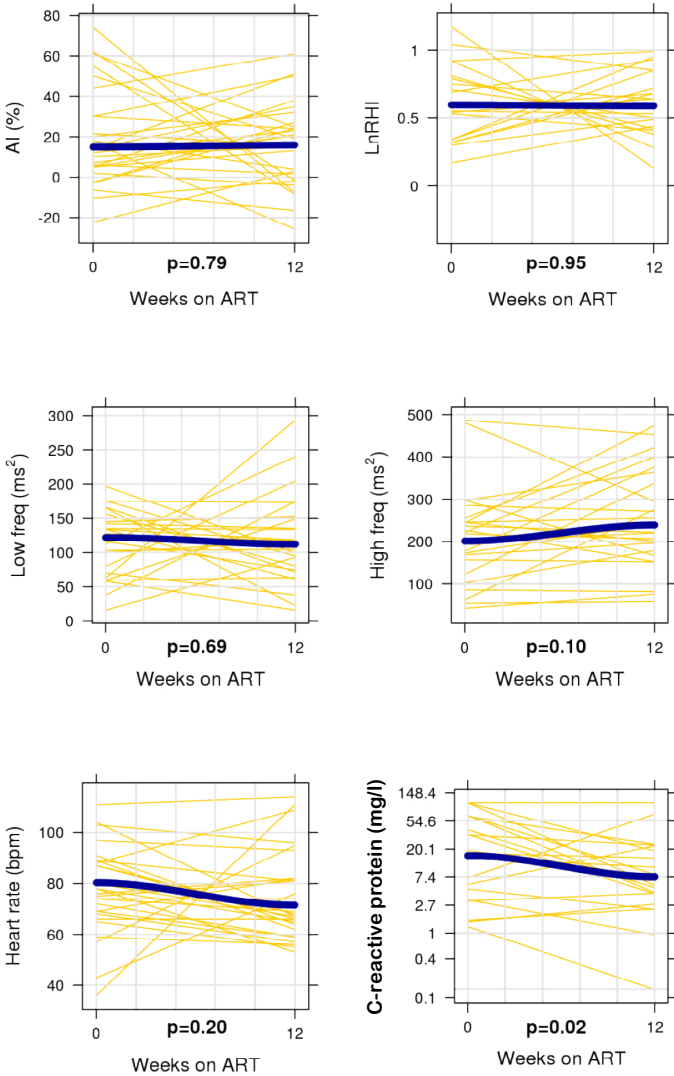
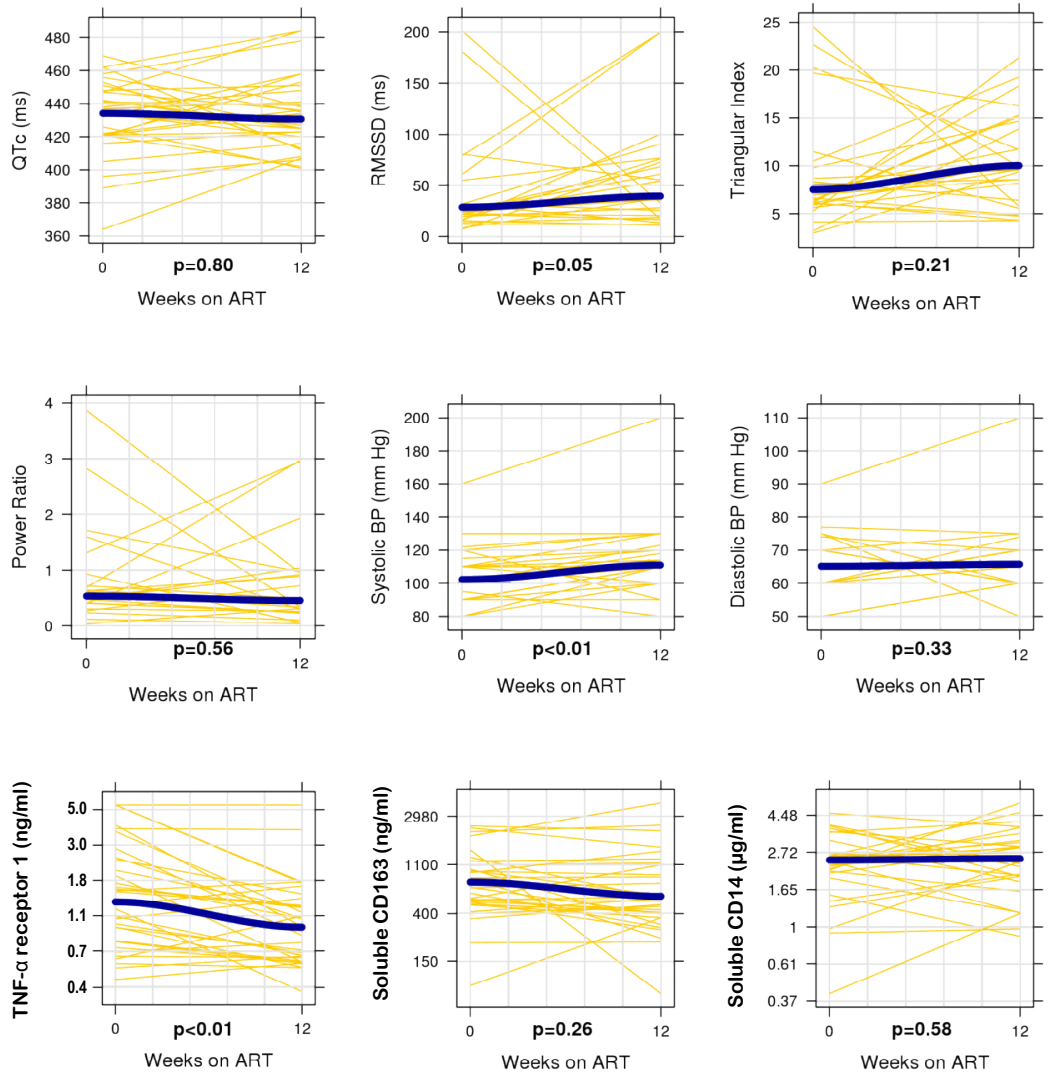

Figure 1: Changes in inflammation biomarkers and cardiovascular parameters over 12 weeks of antiretroviral therapy.

Yellow lines represent paired samples and blue lines represent smoothed (loess) curves fit to the data. As in the statistical models, the inflammation biomarkers are log-transformed and the cardiovascular markers are on the unit scale. Wilcoxon signed rank test was used to assess statistical significance.

higher in HIV patients with elevated CRP (mean CRP $3.3 \mathrm{mg} / \mathrm{l}$ ) when compared to those with normal CRP (mean CRP $1.7 \mathrm{mg} / \mathrm{l}$ ) [24]. One explanation for the high mortality rate among malnourished patients in the early post-ART period may be that increased metabolic activity and weight gain accompanying virologic suppression places sudden strain on a cardiovascular system already injured by the effects of advanced HIV disease and chronic inflammation.
Increases in LnRHI, indicating improved arteriolar responsiveness following ischemia, were associated with reductions in circulating TNF- $\alpha$ R1 levels over 12 weeks, and a similar relationship with CRP levels approached significance $(\mathrm{p}=0.07)$. TNF- $\alpha$ is known to contribute to the pathogenesis of atherosclerosis, heart failure, and myocardial ischemia, and both CRP and TNF- $\alpha$ are associated with markedly elevated coronary artery calcium scores and carotid intima-media 
Citation: Bestawros M, Chidumayo T, Blevins M, Canipe A, Bala J, et al. (2015) Increased Systemic Inflammation is Associated with Cardiac and Vascular Dysfunction over the First 12 Weeks of Antiretroviral Therapy among Undernourished, HIV-Infected Adults in Southern Africa. J AIDS Clin Res 6: 431. doi:10.4172/2155-6113.1000431

thickness, two surrogate markers of subclinical atherosclerosis $[9,25]$. Similarly, LnRHI is significantly associated with carotid intima-media thickness and with coronary artery disease, and it is an independent predictor of major adverse cardiac events [26-28]. The combination of elevated CRP or TNF- $\alpha$ with impaired endothelium-dependent vasodilation correlates with increased risk of cardiac events in patients with coronary artery disease, ischemic cardiomyopathy, nonischemic cardiomyopathy, post-cardiotomy heart failure, and cardiogenic shock [10,29-31]. However, in our pooled analysis, TNF- $\alpha$ R1 levels were positively associated with LnHRI, which was unexpected and may indicate a more complex relationship.

Heart rate varies with inputs from the autonomic nervous system; therefore, heart rate variability has been used to measure autonomic function [32]. In our cohort, higher CRP was significantly associated with reductions in several markers of HRV (RMSSD, triangular index, and high-frequency variations), and TNF- $\alpha$ R1 was closely related with two of these parameters (RMSSD and triangular index). Low HRV is known to be associated with disease severity in chronic coronary artery disease, heart failure, and atrial fibrillation, and with sudden cardiac death among patients with heart failure, atrial fibrillation, or recent myocardial infarction [33-37].

We observed divergent relationships between the inflammation markers and peripheral AI as compared to LnRHI. The median AI value increased in the cohort as a whole over 12 weeks, indicative of increasing arterial stiffness, and greater gains in AI were associated with larger reductions in circulating CD163 over the same period. A prior study of aortic inflammation in HIV-infected adults in the United States using positron emission tomography found greater aortic arterial inflammation was associated with higher circulating CD163 levels, and we had expected that ART initiation would result in reduced vascular monocyte activation and relaxation of the arteries [38]. However, two recent studies suggest that both systemic and vascular inflammation are not major determinants of arterial stiffness as measured by AI $[39,40]$. Furthermore, the median baseline peripheral AI value of 11.9 in our cohort was approximately half the mean value of 21.4 measured using the EndoPAT device in a cohort of healthy black Americans, and the increase in AI we observed may represent a return to a more normal physiologic state on ART rather than a pathologic stiffening of the vasculature related to cardiovascular disease [19]. The inverse CD163-AI relationship in our cohort may be a chance finding, and further studies are needed to investigate the etiology and consequences of low arterial stiffness in malnourished adults with HIV.

Lastly, there was a significant increase in the urine isoprostane/ creatinine ratio over 12 weeks, but the ratio was not associated with any of the inflammatory biomarkers or with the cardiovascular parameters. We attribute this rise in the peroxidation of essential fatty acids to increased free radicals resulting from accelerated metabolic activity on ART, which was not sufficient to exert a significant modulating effect on the already high levels of circulating inflammatory biomarkers [41]. On the other hand, the increase in the urine isoprostane/creatinine ratio could also be due to a reduction in the urine creatinine concentration, either because ART improved nitrogen retention or participants were less dehydrated at 12 weeks as compared to baseline.

Our pilot study was limited by small sample size and high levels of incomplete follow-up; the latter is common in Sub-Saharan African ART programs. Additionally, the study only included undernourished, HIV-infected adults and did not include normal-BMI controls, which precluded an assessment of whether our findings are specific only to undernourished HIV-infected adults or more broadly characterize patients starting ART in Zambia. Lastly, as the goal of this pilot study was to explore potential links between inflammation and cardiovascular instability in a population not previously studied in detail, we performed a large number of statistical comparisons in an exploratory manner without adjusting for multiple comparisons. This may have led to chance findings, such as the conflicting relationships between TNF- $\alpha$ $\mathrm{R} 1$ and LnRHI, and our results need to be confirmed in a large cohort.

\section{Conclusions}

In this pilot study, higher circulating levels of systemic inflammatory biomarkers were associated with several indicators of impaired cardiovascular health in undernourished HIV-infected adults starting ART in Zambia. This may implicate disruptions in cardiac or vascular physiology as one of the causal links in the well-described relationship between persistent inflammation and early mortality in this population. At present there is a paucity of data on cardiovascular disease risk factors among HIV-infected individuals in sub-Saharan Africa. Our findings should be explored in a larger cohort to assess the mortality burden of cardiac events in the first months after starting HIV treatment, to examine underlying biological mechanisms, and to identify opportunities for therapeutic intervention.

\section{Acknowledgements and Funding}

The authors would like to thank Dr. Abdel Sakr for his assistance with the cardiovascular assessments, the NUSTART participants and study staff, and the Zambian Ministry of Health for consistent support of research in the nationa HIV care and treatment program. This work was supported by the Vanderbilt Meharry Center for AIDS Research (NIH grant number P30 AI54999); the NIH Fogarty International Center, Office of the Director, National Institutes of Health, National Heart, Blood, and Lung Institute, and National Institute of Mental Health through the Vanderbilt-Emory-Cornell-Duke Consortium for Global Health Fellows (grant number R25 TW009337); the National Center for Advancing Translational Sciences (CTSA award number UL1TR000445) and the European and Developing Countries Clinical Trials Partnership (grant IP.2009.33011.004).

\section{References}

1. Stringer JS, Zulu I, Levy J, Stringer EM, Mwango A, et al. (2006) Rapid scaleup of antiretroviral therapy at primary care sites in Zambia: feasibility and early outcomes. JAMA 296: 782-793.

2. Koethe JR, Lukusa A, Giganti MJ, Chi BH, Nyirenda CK, et al. (2010) Association between weight gain and clinical outcomes among malnourished adults initiating antiretroviral therapy in Lusaka, Zambia. J Acquir Immune Defic Syndr 53: 507-513.

3. Zachariah R, Fitzgerald M, Massaquoi M, Pasulani O, Arnould L, et al. (2006) Risk factors for high early mortality in patients on antiretroviral treatment in a rural district of Malawi. AIDS 20: 2355-2360.

4. Gupta A, Nadkarni G, Yang WT, Chandrasekhar A, Gupte N, et al. (2011) Early mortality in adults initiating antiretroviral therapy (ART) in low- and middleincome countries (LMIC): a systematic review and meta-analysis. PLoS One 6: e28691.

5. Koethe JR, Blevins M, Nyirenda C, Kabagambe EK, Shepherd BE, et al. (2011) Nutrition and inflammation serum biomarkers are associated with 12-week mortality among malnourished adults initiating antiretroviral therapy in Zambia. J Int AIDS Soc 14: 19.

6. Kuller LH, Tracy R, Belloso W, De Wit S, Drummond F, et al. (2008) Inflammatory and coagulation biomarkers and mortality in patients with HIV infection. PLoS Med 5: e203.

7. Hajiasgharzadeh K, Mirnajafi-Zadeh J, Mani AR (2011) Interleukin-6 impairs chronotropic responsiveness to cholinergic stimulation and decreases heart rate variability in mice. Eur $\mathrm{J}$ Pharmacol 673: 70-77.

8. Ikonomidis I, Stamatelopoulos K, Lekakis J, Vamvakou GD and Kremastinos DT (2008) Inflammatory and non-invasive vascular markers: the multimarker approach for risk stratification in coronary artery disease. Atherosclerosis 199: 3-11.

9. Freitas WM, Quaglia LA, Santos SN, Soares AA, Japiassu AV, et al (2011) Association of systemic inflammatory activity with coronary and carotid atherosclerosis in the very elderly. Atherosclerosis $216: 212-216$. 
Citation: Bestawros M, Chidumayo T, Blevins M, Canipe A, Bala J, et al. (2015) Increased Systemic Inflammation is Associated with Cardiac and Vascular Dysfunction over the First 12 Weeks of Antiretroviral Therapy among Undernourished, HIV-Infected Adults in Southern Africa. J AIDS Clin Res 6: 431. doi:10.4172/2155-6113.1000431

10. Tentolouris C, Tousoulis D, Antoniades C, Bosinakou E, Kotsopoulou M, et al. (2004) Endothelial function and proinflammatory cytokines in patients with ischemic heart disease and dilated cardiomyopathy. International Journal of Cardiology 94: 301-305.

11. Hansson GK (2009) Atherosclerosis--an immune disease: The Anitschkov Lecture 2007. Atherosclerosis 202: 2-10.

12. Tonelli M, Sacks F, Pfeffer M, Gao Z, Curhan G (2005) Relation between serum phosphate level and cardiovascular event rate in people with coronary disease. Circulation 112: 2627-2633.

13. Foley RN (2009) Phosphate levels and cardiovascular disease in the genera population. Clin J Am Soc Nephrol 4: 1136-1139.

14. Venditti FJ, Marotta C, Panezai FR, Oldewurtel HA, Regan TJ (1987) Hypophosphatemia and cardiac arrhythmias. Miner Electrolyte Metab 13: 1925.

15. Schwartz A, Gurman G, Cohen G, Gilutz H, Brill S, et al. (2002) Association between hypophosphatemia and cardiac arrhythmias in the early stages of sepsis. Eur J Intern Med 13: 434.

16. Zazzo JF, Troche G, Ruel P, Maintenant J (1995) High incidence of hypophosphatemia in surgical intensive care patients: efficacy of phosphorus therapy on myocardial function. Intensive Care Med 21: 826-831.

17. Task Force of the European Society of Cardiology and the North American Society of Pacing and Electrophysiology (1996) Heart rate variability: standards of measurement, physiological interpretation and clinical use. Circulation 93 : 1043-1065.

18. Bonetti PO, Pumper GM, Higano ST, Holmes DR, Kuvin JT, et al. (2004) Noninvasive identification of patients with early coronary atherosclerosis by assessment of digital reactive hyperemia. J Am Coll Cardiol 44: 2137-2141.

19. Morris AA, Patel RS, Binongo JN, Poole J, Al Mheid I, et al. (2013) Racial differences in arterial stiffness and microcirculatory function between Black and White Americans. J Am Heart Assoc 2: e002154.

20. Shah SA, Kambur T, Chan C, Herrington DM, Liu K, et al. (2013) Relation of short-term heart rate variability to incident heart failure (from the Multi-Ethnic Study of Atherosclerosis). Am J Cardiol 112: 533-540.

21. Dekker JM, Crow RS, Folsom AR, Hannan PJ, Liao D, et al. (2000) Low heart rate variability in a 2-minute rhythm strip predicts risk of coronary heart disease and mortality from several causes: the ARIC Study. Atherosclerosis Risk In Communities. Circulation 102: 1239-1244.

22. Yeh ET and Willerson JT (2003) Coming of age of C-reactive protein: using inflammation markers in cardiology. Circulation 107: 370-371.

23. Triant VA, Meigs JB, Grinspoon SK (2009) Association of C-reactive protein and HIV infection with acute myocardial infarction. J Acquir Immune Defic Syndr 51: 268-273.

24. Duprez DA, Neuhaus J, Kuller LH, Tracy R, Belloso W, et al. (2012) Inflammation, coagulation and cardiovascular disease in HIV-infected individuals. PLoS One 7: e44454.

25. Kleinbongard P, Heusch G, Schulz R (2010) TNFalpha in atherosclerosis, myocardial ischemia/reperfusion and heart failure. Pharmacol Ther 127: 295314.

26. Fitch KV, Stavrou E, Looby SE, Hemphill L, Jaff MR, et al. (2011) Associations of cardiovascular risk factors with two surrogate markers of subclinical atherosclerosis: endothelial function and carotid intima media thickness. Atherosclerosis 217: 437-440.
27. Kuvin JT, Mammen A, Mooney P, Alsheikh-Ali AA, Karas RH (2007) Assessment of peripheral vascular endothelial function in the ambulatory setting. Vasc Med 12: $13-16$.

28. Rubinshtein R, Kuvin JT, Soffler M, Lennon RJ, Lavi S, et al. (2010) Assessment of endothelial function by non-invasive peripheral arterial tonometry predicts late cardiovascular adverse events. Eur Heart J 31: 1142-1148.

29. Gonzalez MA, Selwyn AP (2003) Endothelial function, inflammation, and prognosis in cardiovascular disease. Am J Med 115 Suppl 8A: 99S-106S.

30. Kang SM, Chung N, Kim JY, Koo BK, Choi D, et al. (2002) Relation of vasodilator response of the brachial artery to inflammatory markers in patients with coronary artery disease. Echocardiography 19: 661-667.

31. Hermansen SE, Kalstad T, How OJ, Myrmel T (2011) Inflammation and reduced endothelial function in the course of severe acute heart failure. Transl Res 157: 117-127.

32. Kleiger RE, Stein PK, Bigger JT (2005) Heart rate variability: measurement and clinical utility. Ann Noninvasive Electrocardiol 10: 88-101.

33. Janszky I, Ericson M, Mittleman MA, Wamala S, Al-Khalili F, et al. (2004) Heart rate variability in long-term risk assessment in middle-aged women with coronary heart disease: The Stockholm Female Coronary Risk Study. J Intern Med 255: 13-21.

34. Kotecha D, New G, Flather MD, Eccleston D, Pepper J, et al. (2012) Fiveminute heart rate variability can predict obstructive angiographic coronary disease. Heart 98: 395-401.

35. Nolan J, Batin PD, Andrews R, Lindsay SJ, Brooksby P, et al. (1998) Prospective study of heart rate variability and mortality in chronic heart failure: results of the United Kingdom heart failure evaluation and assessment of risk trial (UK-heart). Circulation 98: 1510-1516.

36. Lombardi F, Colombo A, Basilico B, Ravaglia R, Garbin M, et al. (2001) Heart rate variability and early recurrence of atrial fibrillation after electrical cardioversion. J Am Coll Cardiol 37: 157-162.

37. Yamada A, Hayano J, Sakata S, Okada A, Mukai S, et al. (2000) Reduced ventricular response irregularity is associated with increased mortality in patients with chronic atrial fibrillation. Circulation 102: 300-306.

38. Subramanian S, Tawakol A, Burdo TH, Abbara S, Wei J, et al. (2012) Arteria inflammation in patients with HIV. JAMA 308: 379-386.

39. Blann AD, Kuzniatsova N, Lip GY (2013) Inflammation does not influence arterial stiffness and pulse-wave velocity in patients with coronary artery disease. J Hum Hypertens 27: 629-634.

40. Heffernan KS, Kuvin JT, Sarnak MJ, Perrone RD, Miskulin DC, et al. (2011) Peripheral augmentation index and vascular inflammation in autosomal dominant polycystic kidney disease. Nephrol Dial Transplant 26: 2515-2521.

41. Morrow JD, Hill KE, Burk RF, Nammour TM, Badr KF, et al. (1990) A series of prostaglandin F2-like compounds are produced in vivo in humans by a noncyclooxygenase, free radical-catalyzed mechanism. Proc Natl Acad Sci U S A 87: 9383-9387. 